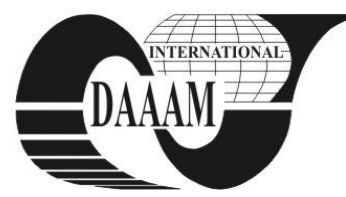

Annals of DAAAM for 2011 \& Proceedings of the 22nd International DAAAM Symposium, Volume 22, No. 1, ISSN 1726-9679 ISBN 978-3-901509-83-4, Editor B. Katalinic, Published by DAAAM International, Vienna, Austria, EU, 2011 Make Harmony between Technology and Nature, and Your Mind will Fly Free as a Bird Annals \& Proceedings of DAAAM International 2011

\title{
DESIGN A PNEUMATIC PARALLEL MANIPULATOR OF TRIPOD TYPE
}

\author{
LASKI, P[awel] A[ndrzej] \& TAKOSOGLU, J[akub] E[manuel]
}

\begin{abstract}
The paper presented the design and experimental test of a tripod type-3UPRR pneumatic parallel manipulator. This manipulator consists of three identical kinematic chains (pneumatic axes) connecting the fixed base and the moving platform. The tool center point TCP of the moving platform is a resultant of relocation of three pneumatic rodless cylinders independently controlled by servo-valves. For simulation purposes a solid model of pneumatic tripod parallel manipulator in SolidWorks was constructed. Since the application of 3-CAD in modelling kinematics and dynamics of parallel manipulators is restricted further simulation was carried out by means of SimMechanics library and MatlabSimulink package. The experimental research focused on determining the precision of positioning of manipulator's endeffector point of the moving platform during point-to-point control.
\end{abstract}

Key words: parallel kinematics, pneumatic robots, pneumatic servo-valve, parallel manipulators

\section{INTRODUCTION}

The development of automation and robotics has increased interest in multi-axis pneumatic systems which are highly dynamic and reliable. However, due to unsatisfactory positioning accuracy of pneumatic servo-cylinder, their application in multi-axis manipulators is restricted. Pneumatic servo-cylinders used in multi-axis electro-pneumatic systems and referred to as pneumatic axes perform opera-tions and function as supporting structure. Cartesian manipulators with pneumatic axes connected in series are classi-fied as open-loop chain kinematic mechanisms. In serial kinematic chain elastic strains accumulate on particular pneumatic axes, which lowers the positioning accuracy of pneumatic manipulators. Parallel mechanism is a closed-loop mechanism in which the moving platform is connected to the fixed base by independent kinematic chains. Kinematic structure in the form of a closedloop chain finds application in parallel kinematic robot and parallel kinematic machine (PKM). Manipulators based on parallel kinematics structure can achieve better accuracy of repeatability and they can apply larger forces than conventional serial manipulators because of the higher stiffness of their mechanical structure.

\section{PROTOTYPE PNEUMATIC PARALLEL MANIPULATOR}

A prototype of pneumatic translational parallel manipulator of tripod kinematic structure was constructed in the Division of Mechatronics (Kielce University of Technology, Poland) (Laski \& Dindorf, 2007). The prototype of tripod parallel manipulator with Festo servo-pneumatic precision positioning systems is presented in Fig. 1. The manipulator possesses a supporting structure, fixed base, moving platform and three pneumatic linear motions (servopneumatic axis). Each servo-pneumatic axis consists of: rodless pneumatic cylinder type DGPIL-25600 (Festo) with integral feedback transducer (built-in
Temposonic encoders for continual positioning feedback to the master control unit), 5/3 servopneumatic valve (directional proportional control valve) type MPYE-5-1/8-HF-010B (Festo), axis interface type SPC-AIF, positioning axis sub-controller type SPC-200 (the use of a sub-controller card permits control of up to four axes) and Ethernet/Can Bus interface (Festo). Kinematic structure and structure control of the prototype of 3DoF pneumatic translational parallel manipulators is shown in Fig.2.

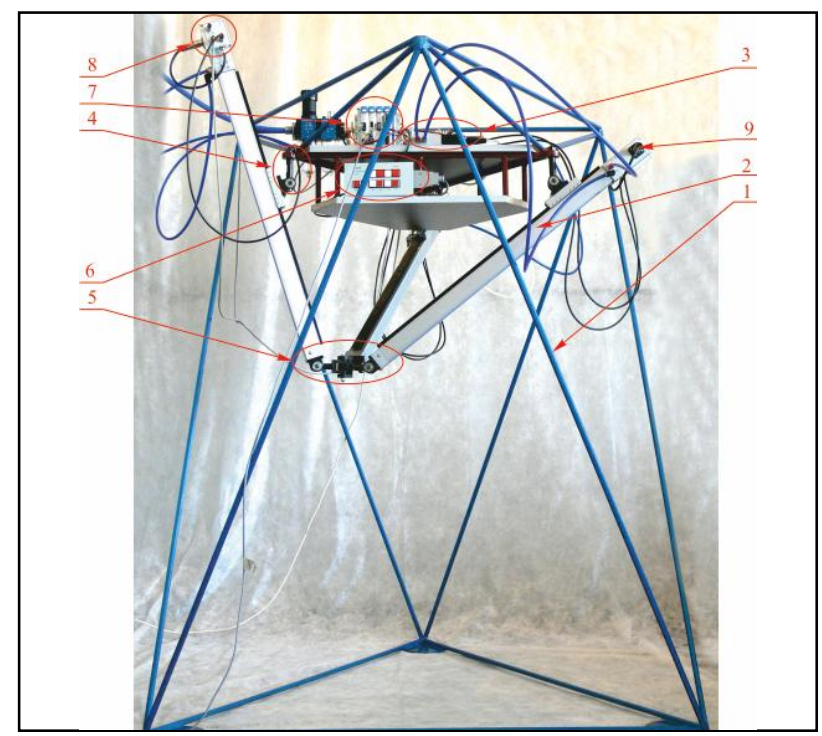

Fig. 1. Prototype of 3-UPRR pneumatic parallel manipulator: 1 - supporting structure, 2 - rodless pneumatic cylinder, 3 proportional directional control valve, 4 - universal Cardan joint, 5 - moving platform, 6 - control panel, 7 - positioning axis controller, 8 - axis interface, 9 - axis connector

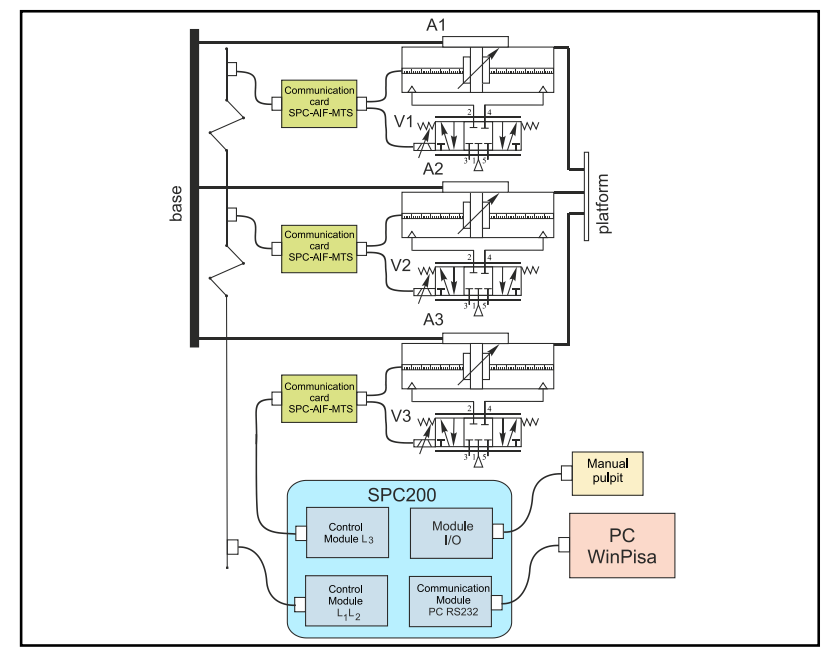

Fig. 2. Structure control of 3-UPRR pneumatic parallel manipulator 
Each of the three identical closed-loop chains of the manipulator consists of serial kinematic chains: universal cardan joint $(\mathrm{U})$, prismatic joint $(\mathrm{P})$, formed by a rodless pneumatic cylinder and two revolute joints $(2 \mathrm{R})$ formed after universal cardan had been parted. The slide of rodless cylinder was connected with fixed base by means of articulated joints of $\mathrm{U}$ cardan and the end cap of cylinder were connected by revolute joint $\mathrm{R}$ to the moving platform. The second revolute joint $\mathrm{R}$ was placed in tool center point (TCP) of the moving platform. The presented construction of the parallel manipulator ensures parallel position of the moving platform to the fixed base for optional posi-tion of pneumatic cylinder. The kinematic structure of a new prototype of 3-UPRR pneumatic parallel manipulator is an interesting solution expanding the architecture of parallel manipulators, type 3-DoF TPM.

\section{MODEL RESEARCH ON PNEUMATIC PARALLEL MANIPULATOR}

Software - CAD commonly used by constructors enables de-signing solid models of complex mechanisms of parallel kinematics. A solid model of 3-UPRR pneumatic parallel manipulator obtained by SolidWorks is presented in Fig. 3.

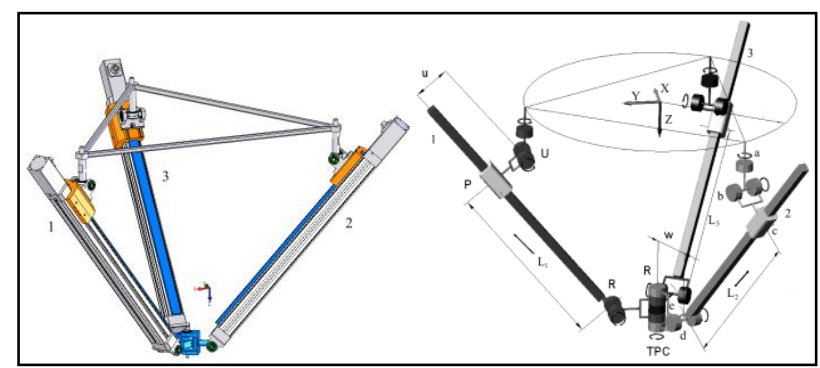

Fig. 3. Solid and kinematic model of 3-UPRR pneumatic parallel manipulator

A revolute joint $\mathrm{R}$ is connecting the end cap of pneumatic cylinders with moving platform in point TCP and a $\mathrm{U}$ joint is connecting the slide pneumatic cylinder with a fixed base. In order to record geometric and kinematic relations holding for pneumatic parallel manipulator of 3-UPRR kinematics, its kinematic model presented in Fig. 3 (Takosoglu et al., 2010). By means of Dynamic Designer Motion, which possesses graphic interface SolidWorks the simulation of pneumatic parallel manipulator's motion was conducted. In order to simulate the manipulator's motion it was necessary to define the basic parameters, kinematic joints and motion restrictions. For solid model a few composite relations were defined which enabled assigning them kinematic joints.

The solution of manipulator's kinematic reverse problem used in the design of control algorithm enabled to determine lengths of the movable links L1,L2.L3 (displacement of linear pneumatic actuators) in relation to the coordinates of endeffector point $\mathrm{P}(\mathrm{x}, \mathrm{y}, \mathrm{z})$ of tripod parallel manipulator:

$$
\begin{aligned}
& L_{1}=\sqrt{z^{2}-U^{2}+\left(\sqrt{(x-r)^{2}+y^{2}}-W\right)^{2}} \\
& L_{2}=\sqrt{z^{2}-U^{2}+\left(\sqrt{\left(x+r \cdot \frac{1}{2}\right)^{2}+\left(y+r \cdot \frac{\sqrt{3}}{2}\right)^{2}}-W\right)^{2}} \\
& L_{3}=\sqrt{z^{2}-U^{2}+\left(\sqrt{\left(x+r \cdot \frac{1}{2}\right)^{2}+\left(y-r \cdot \frac{\sqrt{3}}{2}\right)^{2}}-W\right)^{2}}
\end{aligned}
$$

where: $\mathrm{x}, \mathrm{y}, \mathrm{z}$ - coordinates of end-effector point; $\mathrm{P}, \mathrm{r}$ - ray of the circle circumscribing the equilateral triangle at the point where pneumatic servo-motors are fixed; $\mathrm{z}, \mathrm{U}, \mathrm{W}$ - geometric dimensions of the manipulator for the initial position of $\mathrm{P}$ point for the pneumatic actuators at their maximum stroke; $\mathrm{L}_{1}, \mathrm{~L}_{2} \cdot \mathrm{L}_{3}$ lengths of the movable links.

Since the application of SolidWorks in modeling kinematics and dynamics of parallel manipulators is restricted, further simulation was carried out by means of SimMechanics library of Matlab-Simulink package. The library enables the construction of complex mechanisms of parallel manipulators excluding mathematical descriptions of their kinematics and dynamics. In simulations based upon SimMechanics library an equivalent model of pneumatic tripod manipulator with its spatial orientation indicated was constructed. In SimMechanics library all the solid elements of the manipulator were described by substitute geometry by means of ellipsoids and assigned both masses and inertial tensors. In Matlab-Simulink environment tripod-based parallel kinematic manipulator was connected with its control system.

\section{CONCLUSION}

The paper presents the prototype of tripod pneumatic parallel manipulator of 3-UPRR kinematic structure. The tripod pneumatic manipulator consists of a supporting structure, fixed base, moving platform, prismatic joints (P-joints) - three servopneumatic axes, Cardan joints (U-joints) and rotary joints (Rjoints). The single servo-pneumatic axis of the manipulator is composed of rodless pneumatic cylinder integrated with magnetostrictive measurement of position, proportional directional control valve, axis interface and axis connector, positioning axis controller (Dzierzek 2007). The aim of the project was to carry out model tests and simulations on a virtual model of tripod manipulator as well as conduct experimental research on a prototype of pneumatic parallel manipulator of 3UPRR kinematics. Both the model tests and the experimental results show that the pneumatic parallel manipulator of 3UPRR type fulfills the constructional assumptions and has satisfactory kinematic and dynamic properties. Further research will be directed towards improving the control accuracy of endeffector's trajectory by means of fuzzy logic control system (FLC) with real time interpolator and dSPACE software (Takosoglu et al., 2009). The research conducted on the prototype of the pneumatic parallel manipulator of tripod kinematic structure is an original contribution towards development of parallel kinematic structures of pneumatic manipulators. The presented prototype of pneumatic parallel manipulator will find its application in manufacturing manipulators and rehabilitation manipulators (Siemieniako et al., 2009).

\section{REFERENCES}

Dzierzek, K.; (2009). The digital system of the position measurement, Solid State Phenomena, Vol. 147-149, 936941, ISSN: 1662-9779

Laski, P.; Dindorf, R. (2007). Prototype of pneumatic parallel manipulator, Hydraulika a Pneumatika (Slovak Republic), No 1, 22-24, ISSN: 1335-5171

Siemieniako, F.; Ostaszewski, M.; Kuzmierowski, T. (2010). Analysis of requirements for exoskeleton construction, International Journal of Applied Mechanics and Engineering, Vol.15, No 3, 841-846, ISSN: 1425-1655

Takosoglu, J. E.; Dindorf, R. F.; Laski, P. A. (2009). Rapid prototyping of fuzzy controller pneumatic servo-system, International Journal of Advanced Manufacturing Technology, Vol. 40, No. 3-4, 01.2009 349-361, ISSN: 0268-3768

Takosoglu, J. E.; Dindorf, R. F.; Laski, P. A. (2010). Fuzzy logic positioning system of electro-pneumatic servo-drive, In: Robot Manipulators, Trends and Development, Jimenez A., Al Hadithi B. M., 298-320, In-Tech, ISBN 978-953307-073-5, Croatia 